\title{
The Relationship of Aerobic Endurance and Linear Speed on Repeat Sprint Ability Performance in Female International Footballers
}

\author{
Brian Doyle $^{1, *}$, Declan Browne ${ }^{1}$, Dan Horan ${ }^{2}$ \\ ${ }^{1}$ Department of Science and Health, Institute of Technology, Carlow, Ireland \\ ${ }^{2}$ Department of High Performance, Football Association of Ireland, Abbottstown, Dublin, Ireland
}

Received July 7, 2020; Revised August 18, 2020; Accepted August 28, 2020

\section{Cite This Paper in the following Citation Styles}

(a): [1] Brian Doyle, Declan Browne, Dan Horan, "The Relationship of Aerobic Endurance and Linear Speed on Repeat Sprint Ability Performance in Female International Footballers, "International Journal of Human Movement and Sports Sciences, Vol. 8, No. 4, pp. 147 - 153, 2020. DOI: 10.13189/saj.2020.080407.

(b): Brian Doyle, Declan Browne, Dan Horan (2020). The Relationship of Aerobic Endurance and Linear Speed on Repeat Sprint Ability Performance in Female International Footballers. International Journal of Human Movement and Sports Sciences, 8(4), 147 - 153. DOI: 10.13189/saj.2020.080407.

Copyright $(2020$ by authors, all rights reserved. Authors agree that this article remains permanently open access under the terms of the Creative Commons Attribution License 4.0 International License

\begin{abstract}
The aim of this study was to investigate the relationship between aerobic endurance and linear speed on repeat sprint ability performance in female international footballers. Twenty-five female international football players completed 10-m, 20-m, 30-m linear speed, repeat sprint ability (RSA) and yo-yo intermittent recovery, level 1 tests. Pearson's product correlation (r) was used to assess the relationship between $10-\mathrm{m}, 20-\mathrm{m}$ and $30-\mathrm{m}$ sprint times (S10-m, S20-m, S30-m) sprint time on the first trial of the repeated sprint test (RST1) and yo - yo intermittent recovery test, level 1 (YYIR1) with total repeated sprint time (RSTT). Linear regressions were used to describe the relationship between the dependent variable (RSTT) and independent variables (S10-m, S20-m, S30-m, RST1 and YYIR1). Pearson's correlation analysis indicated a large negative correlation between RSTT and YYIR1 performance $(r=-0.58, P=.002)$, a very large significant relationship was established between S10-m and S20-m whilst an almost perfect relationship was present for S30-m and RST1. The results of the linear regression proved the independent variable RST1 explained $85 \%$ (adjusted $\mathrm{R}^{2}$ ) of the variation in RSTT scores. S10-m, S20-m and S30-m performance accounted for $62-83 \%$ of the variation in RSTT scores whilst YYIR1 performance only accounted for $33.6 \%$. The results of this study indicate that fast linear speed can positively impact on RSTT performance whilst reduced aerobic endurance capabilities may negatively
\end{abstract}

influence RSTT performance in female international footballers.

Keywords Sprinting, Endurance, Fatigue, Female, Football

\section{Introduction}

Elite female international football is a dynamic intermittent field sport that physiologically stresses the metabolic and neuromuscular systems $[1,2]$. Due to the random nature of football match play numerous contextual factors will influence the physical demands withstood by players such as playing position, formation, tactics, time in possession, team ranking and score line [3-5]. Players transition from long periods of aerobic activity to short bouts of high intensity anaerobic activities such as high speed running, sprinting and repeated high speed and sprinting actions [6-8].

During match play total distances covered fall within the range of $9-12 \mathrm{~km}$ with the majority of distance covered walking or jogging with high speed running $(>18 \mathrm{~km}-\mathrm{h})$ been shown to account for $1.53-1.68 \mathrm{~km}$ of that total distance $[7,5,9,10]$. Total match play sprint distance reported within the literature differs due to the variation of 
the sprint speed threshold implemented $(19.1 \mathrm{~km}-\mathrm{h}$ versus $20 \mathrm{~km} . \mathrm{h}$ versus $25.1 \mathrm{~km} . \mathrm{h}$ ) [6,11,12]. Though players have been shown to cover mean distances in the range of .16 - . $.46 \mathrm{~km}$ sprinting during games and complete between 180 and 240 accelerations during a senior international match $[5,9,10,112,13]$. Repeated high speed actions consisting of two or more sprints with less than 20 seconds recovery between efforts occur approximately $31-33$ times during international match play [6,8]. Although, it is not uncommon for players to perform up to 6 repeated high speed bouts with less than 20 second recovery between each bout $[6,8,11]$. However, the most frequent mean recovery time duration between repeated high speed running bouts in female international matches has been demonstrated to be 10 seconds or less [6]. Such repeated high speed and sprint efforts with minimal recovery time can result in a deterioration of high speed running, sprint and RSA performance, throughout international match-play $[5,8,9,14]$.

RSA has been defined within the literature as short sprints typically less than ten seconds in duration with recovery periods sixty seconds or less [15]. Assessment of RSA identifies a player's capacity to maintain maximal effort and recovery during multiple successive high speed or sprint efforts a key attribute for athletes in field based team sports such as football [16]. Numerous assessment methods have been utilised to measure RSA performance in various populations of female football players such as 5 x 10-m Ajax sprint test, 7 x 30-m sprints with 30 seconds recovery, $10 \times 30$-m with 30 seconds recovery and $6 \times 15$ second cycles of $20-\mathrm{m}$ maximal sprinting followed by a $10-\mathrm{m}$ deceleration and a 10-m active recovery jog (Figure 1) making direct comparisons between studies challenging [17-21].

RSA performance is highly influenced by the testing protocol utilised (i.e. sprint duration, distance and number of repetitions as well as type of and duration of recovery) therefore any RSA assessment protocol should be reflective of the movement demands of the sport [16]. In addition, it must be noted that variables sprint duration, distance, number of repetitions and duration of recovery during match play will differ between playing positions $[6,11]$. Spencer et al [17] stated that mean sprint times and distances covered in field based team sports to be $2-3$ seconds in duration and between $10-20 \mathrm{~m}$., findings supported by Gabbett et al [22] demonstrating mean sprint times of $(2.1 \pm 0.7$ seconds $)$. More recently Nakamura et al [11] highlighted similar results reporting mean sprint times of ( $2.5 \pm 0.5$ seconds) and mean sprint distances of (15.7 \pm $2.6 \mathrm{~m})$ in elite female footballers. Furthermore, the recovery period between 2 repeat high speed and sprint bouts the most frequent in female international football has been shown to be $(9.42 \pm 2.68$ and $10.22 \pm 4.42$ seconds $)$ respectively [6,8]. In addition, RSA assessment incorporating active recovery opposed to passive recovery may potentially reduce muscle acidosis and negate a decline in RSA performances [17]. Considering $85 \%$ of the total distance covered during elite female match play is at walking and jogging speeds and approximately $95 \%$ of the activity between repeat high speed or sprint bouts is active in nature RSA assessment should include active recovery between each repeat sprint $[9,22]$.

Similar to Lockie et al [23] this study will implement the RSA assessment protocol developed by Gabbet [21] for elite female footballers (see figure 1) as based on the presented literature the protocol appears to specifically address the RSA demands of elite female football match play. Gabbet [21] previously stated that RSA tests should be performed in conjunction with a maximal aerobic endurance and linear speed assessments. As a highly developed aerobic endurance capacity may benefit recovery between repeated high speed or sprint bouts during match play [19]. Therefore, the purpose of this study was to undertake the hypothesis stated by Gabbet [21] and examine the relationship of aerobic endurance (YYIR1) and linear speed (S10-m, S20-m, S-30m and RST1) on RSA performance in female international footballers

\section{Materials \& Methods}

\subsection{Experimental Approach to the Problem}

Physical assessments were conducted in the following order 10-m, 20-m, 30-m linear sprints, RSA and Yo-Yo Intermittent Recovery Test, level 1 (YYIR1). Assessments took place in two separate testing sessions on the first day of each squads relevant training camp during 2019. All tests were performed in the afternoon between the hours of (12:00 and 16:00). Subjects were provided at least a 15-minute recovery period between tests to assuage any effects of fatigue. Every test was performed indoors on a rigid surface with all subjects wearing running shoes. Prior to the initiation of the testing session, participants underwent a structured 10 minute warm up protocol consisting of jogging, running, lower limb mobility and dynamic stretching movements. On completion of the warm up a 5-minute recovery period was employed to assuage any effects of fatigue. Any participants diagnosed with a lower limb injury were excluded from testing.

\subsection{Participants}

Twenty-five female elite level football players selected to represent the Ireland $\mathrm{u} 19$ or senior international team participated in the study (mean $\pm \mathrm{SD}$; age $19.1+3.4$ years; height $167+6 \mathrm{~cm}$; mass $62.5+7.4 \mathrm{~kg}$ ). Ethical approval was obtained by the college Research Ethics Committee. The participants were informed of the risks of the study in person and writing. An informed consent document was signed before the beginning of data collection and participants were free to withdraw from the study at any 
time. For those under the age of 18 years' age parental or guardian signed consent was obtained. The study was conducted in accordance to the Declaration of Helsinki.

\subsection{Linear Speed}

Speed was assessed over 10-m, 20-m, 30-m using photocell timing gates (Witty-gate, Microgate, Italy) equipment which has been deemed to be a reliable assessor of linear speed in female footballer players [24]. Participants started $0.5 \mathrm{~m}$ behind the initial timing gate in a two point split stance and were instructed to set off in their own time and run maximally to a marker place $2-\mathrm{m}$ beyond the $30-\mathrm{m}$ timing gate. Each subject performed 2 sub maximal efforts sprints prior to 3 maximal efforts separated with a minimum of 2 minutes of rest but no longer than 3 minutes. Times were recorded to the nearest 0.01 with the fastest time of the three efforts at $10-\mathrm{m}, 20-\mathrm{m}$, and $30-\mathrm{m}$ used for analysis. ICC and CVs for 10-m ,20-m and 30-m linear speed assessments fell between the range of (ICC $=.90-.98, \mathrm{CV}=1-2.4 \%$ ).

\subsubsection{Repeat Sprint Ability}

As stated the RSA test protocol developed by Gabbet [21] was used for this study. The test has been shown to be a valid and reliable assessment of RSA in elite female football players $(\mathrm{ICC}=.91, \mathrm{TE}=1.5 \%)$ [21]. Players performed $6 \times 20$-m maximal effort sprints on a 15 - second cycle. On the completion of each timed 20-m sprint, players performed a $10-\mathrm{m}$ deceleration followed by a $10-\mathrm{m}$ active recovery jog back to the next start line before coming to a complete standstill prior to next sprint (Figure 1) [21]. Subjects began their sprint $0.5 \mathrm{~m}$ behind the relevant starting timing gate. Photocell timing gates (Witty-gate, Microgate, Italy) were positioned at the 10-m and $30-\mathrm{m}$ start/finish lines to time each sprint effort which was recorded to the nearest 0.01 second. The researcher recorded the active recovery period between $20-\mathrm{m}$ sprints using a stopwatch (TIS Pro 018, Precision, Nottingham, UK) and provided feedback on the progress of the active recovery period [23]. Each participant performed a single submaximal effort of one 15-second cycle for test protocol familiarisation. Numerous variables were calculated for the RSA test to assess the group's performance and rate of fatigue:

- $\quad$ RSTT $=$ the sum of all six 20-m sprint times stated in seconds $[21,23]$.

- $\quad$ RST1 = the time taken to complete the first $20-\mathrm{m}$ sprint also expressed in seconds [21].

- $\quad F I=$ the decrease in sprint performance from the first to the last sprint was calculated and presented as a percentage. The following equation was used: Fatigue index $(\%)=100 \mathrm{X}$ (fastest sprint - slowest sprint / fastest sprint $[21,23]$.

- $\operatorname{Sdec} \%=$ sprint decrement (\%) calculates fatigue by comparing the actual performance against the ideal performance (i.e. should the best 20 -m effort be replicated in each of the 6 repetitions). The following equation was used: Sprint decrement (\%) = $\{(\mathrm{S} 1+\mathrm{S} 2+\mathrm{S} 3+\mathrm{S} 4+\mathrm{S} 5+\mathrm{S} 6$ / Sbest x 6) -1 $\}$ X 100 [14]

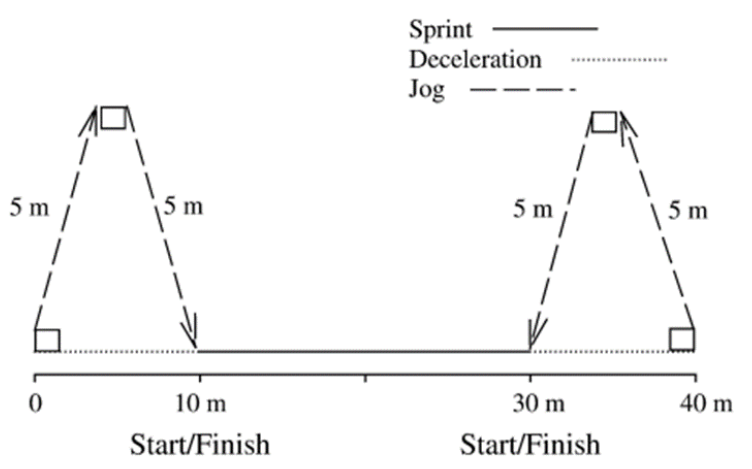

Figure 1. Repeat sprint ability test (Gabbett 2010)

\subsubsection{Aerobic Endurance}

The subjects completed the YYIR1 test as described by Krustrup et al [25] which has been deemed to be a reliable assessment of aerobic endurance [26]. The test was performed using an audio recording. Each athlete performed repeated $20 \mathrm{~m}$ shuttle runs at increasing velocities with 10 seconds of active recovery. During the active recovery participants walked around a marker placed 5-m behind the finishing line. An individual's test was terminated when the subject failed to reach the starting line within the allotted time period on two occasions or the participant felt unable to complete another shuttle at the assigned speed. The total distance covered by a subject was used as the performance measure.

\subsection{Statistical Analysis}

Assumption for normality was confirmed using the Shapiro-Wilks test. Levenes test indicated equal variance to be assumed. Pearson's product moment-correlation (r) was conducted to determine the relationship between 10-m, 20-m and 30-m sprint times, sprint time on the first trial of the repeated sprint test (RST1), yo - yo intermittent recovery test, level 1 (YYIR1) and total repeated sprint time (RSTT) at significance level of $(\mathrm{P}<0.01)$. Strength of the correlation was assigned by the following parameters $0.1-0.3$ (small), $0.3-0.5$ (medium) $0.5-0.7$ (large), 0.7 0.9 (very large) and $0.9-1.0$ (almost perfect) [26]. YYIR1 scores were separated in to high and low performing groups were independent samples t-test analysed differences between the groups. To determine the magnitude of difference between the high and low groups effect sizes (ES: Cohens $d$ ) were calculated and values of $0.2,0.5$, and 0.8 were interpreted as a small, medium and large respectively [28]. Linear regressions were utilised to explain the relationship between the dependent variable (repeat sprint ability total time) and independent variables 
(10-m,20-m,30-m linear sprints, initial 20-m repeat sprint trial and yo-yo intermittent recovery test). The Durban Watson test was applied in the residual of regression analysis. Furthermore, the analysis of variance (ANOVA) test allowed to determine if the linear regression tests were significant.

\section{Results}

Descriptive statistics for all performance test are presented in (Table 1). Pearson product correlation (r) analysis identified a large significant relationship existed between YYIR1 and RSTT $(r=-0.58, P=0.002)$ whilst significant medium to large negative relationships were present with S10-m $(r=-0.42, P=0.038), S 20-m(r=-0.52, P=0.008)$, S30-m $(r=-0.50, P=0.011)$, RST1 $(r=-0.58, P=0.03)$ and YYIR1. Very large significant relationships were established between S10-m $(r=0.78, P=0.0001)$ and S20-m $(r=0.89, P=0.0001)$ with RSTT. In addition, an almost perfect relationship was reveled between $\mathrm{S} 30-\mathrm{m}(\mathrm{r}=$ $0.92, \mathrm{P}=0.0001)$ and RST1 $(\mathrm{r}=0.92, \mathrm{P}=0.0001)$ with RSTT. In terms of rate of fatigue there was no significant relationship established between either FI or Sdec and RSTT. (table 2).

Table 1. Descriptive statistics of Repeat sprint ability, Linear speed and Aerobic endurance tests (mean \pm SD)

\begin{tabular}{|c|c|c|c|c|}
\hline Test & $\mathrm{N}$ & Mean + SD & \multicolumn{2}{|c|}{ CI 95\% } \\
\hline & & & Lower & Upper \\
\hline RSTT (seconds) & 25 & $21.42 \pm .97$ & 21.02 & 21.82 \\
\hline RST1 (seconds) & 25 & $3.43 \pm .16$ & 3.37 & 3.5 \\
\hline FI (\%) & 25 & $8.34 \pm 2.55$ & 7.28 & 9.39 \\
\hline Sdec (\%) & 25 & $4.38 \pm .26$ & 3.84 & 4.93 \\
\hline YYIR1 (m) & 25 & $1080 \pm 375$ & 925 & 1235 \\
\hline $10-m$ (seconds) & 25 & $1.92 \pm .09$ & 1.88 & 1.96 \\
\hline $20-m$ (seconds) & 25 & $3.31 \pm .15$ & 3.25 & 3.37 \\
\hline $30-m$ (seconds) & 25 & $4.62 \pm .21$ & 4.53 & 4.71 \\
\hline
\end{tabular}

Analysis of YYIR1 results indicated the high performing group to significantly cover more distance during the YYIR1 $(1345 \pm 260 \mathrm{~m}$ vs $741 \pm 162 \mathrm{~m}, \mathrm{P}=0.0001, \mathrm{ES}=$ 2.27), performed faster RSTT ( $21.07 \pm 0.80$ vs $21.87 \pm 1.0$ seconds, $\mathrm{P}=0.036, \mathrm{ES}=0.88)$ and $\mathrm{RST} 1$ times $(3.37 \pm$ 0.13 vs $3.51 \pm .17$ seconds, $\mathrm{P}=0.029$, ES $=0.94)$. No statistically significant differences were reported between the high and low groups for S10-m, S20-m and S30-m assessment though moderate effect sizes $(\mathrm{ES}=0.56,0.63$, and 0.58) in favor of the high YYIR1 group were present for all distances. In terms of levels of FI ( $7.92 \pm 2.7$ vs 8.86 $\pm 2.38 \%, \mathrm{ES}=0.37)$ and $\mathrm{Sdec} \%(4.48 \pm 1.56$ vs $4.26 \pm 1 \%$, $\mathrm{ES}=0.16)$ sustained during RSA test small to trivial differences existed between the high and low YYIR1 groups. Linear regression analysis and predicative equations are presented in (table 2).

Table 2. Linear regression data and predictive equations for RSTT versus all independent variables

\begin{tabular}{|c|c|c|}
\hline $\begin{array}{c}\text { Independent } \\
\text { variables }\end{array}$ & $\mathbf{R 2}$ & Predictive Equation \\
\hline YYIR1 & $33.60 \%$ & RSTT $=23.03+-.001$ "YYIR1 distance" \\
\hline S10-m & $62.10 \%$ & RSTT $=6.422+7.804$ "S10m" \\
\hline S20-m & $79.90 \%$ & RSTT $=2.203+5.81$ " S20 m" \\
\hline S30-m & $83.80 \%$ & RSTT $=1.997+4.203$ "30m" \\
\hline RST1 & $85.40 \%$ & RSTT $=2.224+5.590$ "RST1" \\
\hline
\end{tabular}

\section{Discussion}

This study is the first of its kind to investigate the relationship between aerobic endurance (YYIR1) and linear speed (S10-m, S20-m and S30-m) on RSA (RSTT and RST1) performance in female international footballers. The RSTT for the female international football players in this study $(21.42 \pm .97$ seconds) was slower than the national level players $(20.09 \pm .5$ seconds $)$ evaluated by Gabbett [21] but quicker than state $(23.3 \pm .04$ seconds $)$ and collegiate level players (22.09 \pm .99 seconds) [21,23]. The fatigue index also termed performance decrement (PD) for the players in this study $(8.34 \pm 2.5 \%)$ was greater in comparison to that of the national $(6.2 \pm 1.6 \%)$ and state level $(6.8 \pm 2.7 \%)$ players examined by Gabbet [21] but less than the collegiate players $(8.37 \pm 5.51 \%)$ investigated by Lockie et al [23]. In addition, this study was the first to describe the Sdec $(4.38 \pm .26 \%)$ in female international football players using this RSA test protocol. The Sdec method has been shown to the most valid and reliable method of measuring fatigue in RSA assessment as it accounts for all sprints performed opposed to FI or PD which only considers the first and last sprint a scenario which may be considerably influenced by one good or bad repetition during a RSA assessment [29]. However, in this cohort Sdec showed no significant relationship with RSTT $(\mathrm{r}=0.38, \mathrm{P}=.19)$. Future studies should further investigate if $\mathrm{Sdec}$ is a more reliable measure of fatigue during RSA assessment of elite female football players as both FI or PD have previously been shown not to be in national, state and collegiate level football players as well as the cohort in this current investigation $(\mathrm{r}=0.29 \mathrm{P}=.17)[21,23]$.

Aerobic endurance would appear to play a significant role in RSA performance as a large negative correlation highlighted players that covered greater distances in the YYIR1 achieved faster RSTT times (Fig 2). Although the test protocols differed the results of this study would concur with Shalfawi et al [19] findings which reported similar large negative correlations $(\mathrm{r}=-0.59, \mathrm{P}=<0.01)$ between RSTT $(10 \times 30-\mathrm{m}$ with 30 seconds recovery between reps) and aerobic fitness (multi stage fitness test) 
in elite female club level footballers. In contrast Lockie et al [23] using the same test protocols as this study reported no significant relationship between RSTT and YYIR1 performance $(r=-0.03, P=0.91)$. Furthermore, the presence of significant medium to large negative relationships between YYIR1 and S10-m, S20-m, S30-m and RST1 replicates a trend previously established in elite female club level players [19]. Similar to Sanders et al [20] findings on female colligate level football players $30-\mathrm{m}$ sprint performance it would appear that the players in this study who possessed higher aerobic endurance capabilities on average were faster over S10-m, S20-m and S30-m distances.

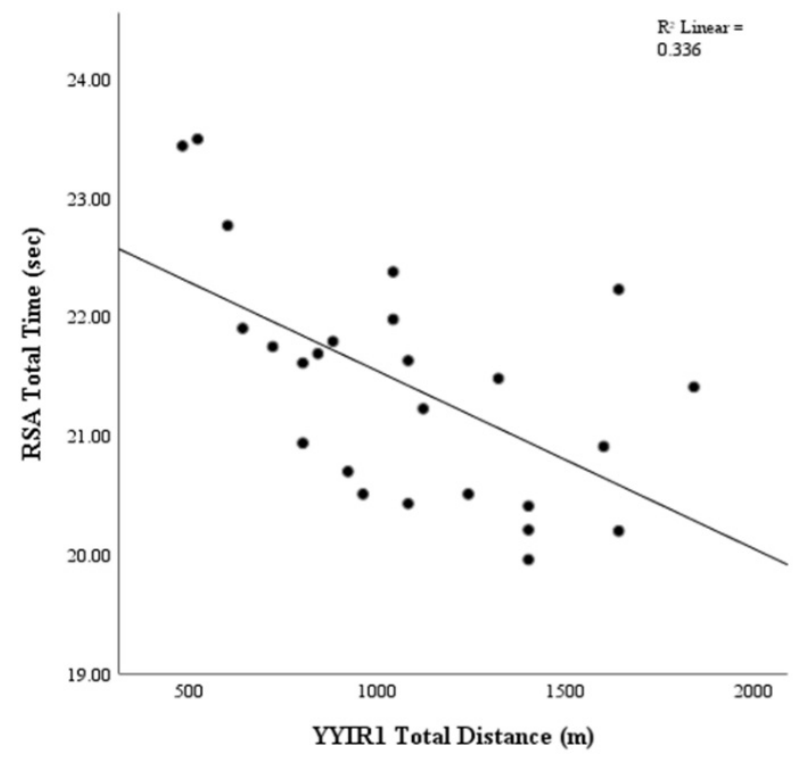

Figure 2. Scatterplot and line of best fit for the relationship between YYIR1 and RSTT

A growing body of research emanating from numerous populations of female football players indicates a strong relationship between linear speed performance over various distances and $\operatorname{RSTT}[19,20,23]$. Contrary to Gabbett [21] $(\mathrm{r}=0.02, \mathrm{P}<0.05)$ this study found a very large significant relationship between S10-m and RSTT performance which was greater than the significantly large relationship previously reported by Lockie et al [23] in collegiate level players $(\mathrm{r}=0.50, \mathrm{P}=0.03)$. Over the longer distances of $0-20-\mathrm{m}$ and $0-30-\mathrm{m}$ this current study as well as Shalfawi et al [19] $(\mathrm{r}=0.73, \mathrm{P}<0.01)$ both highlighted very large significant relationship between S20-m performance and RSTT. While Lockie et al [23] displayed a similar trend between $30-\mathrm{m}$ and RSTT performance $(r=0.71, P<0.01)$ which was further supported by the research findings ofthis study which highlighted an almost perfect relationship. Furthermore, akin to Gabbet $(\mathrm{r}=0.96, \mathrm{P}<0.001)$ an almost perfect significant relationship $(\mathrm{r}=0.92, \mathrm{P}<0.01)$ was present between RST1 and RSTT performance in this cohort of 1 players [21]. Linear regression analysis revealed RST1 was the most strongly correlated with RSTT accounting for $85 \%$ variance in comparison to S10-m, S20-m and S30-m which accounted for $62.1 \%, 79.9 \%$ and $83.8 \%$ variance in RSTT performances. YYIR1 was deemed to have a more moderate influence on RSTT in comparison to all linear speed assessments with the aerobic endurance assessment being responsible for $33.6 \%$ of the variance in RSTT.

\section{Conclusions}

There are a number of limitations to this investigation that should be addressed. The sample size of this study is a combination of two international age groups (U19 and senior) therefore the generalisation of these results must be interpreted with caution. Future RSA research should specifically examine all competitive international age categories (i.e., U17, U19 and senior). In addition, no physiological markers such as heart-rate [21] were assessed during the RSA test which may have been of benefit. However, significant relationships were observed between aerobic endurance and linear speed with RSA performance. The results from this study indicate a players RSA will be influenced by the inter-play of both their aerobic endurance and linear speed capabilities opposed to either one independently. Therefore, it may be suggested that separate training strategies for aerobic endurance and linear speed are required to improve a player's RSA.

\section{Practical Application}

In order to fully optimise RSA performance of elite female footballer's coaches, strength and conditioning coaches and sports scientist alike should implement a concurrent training strategy. For instance, small sided games are common practice in the development of female football player's aerobic capacity though they have been shown not to meet the high speed, very high speed and sprinting demands of international match play [30]. One may suggest that aerobic endurance development can be targeted via various sized small sided games whilst linear speed development can be addressed through sprint training and power development. Though it must be noted that any training program designed to optimise RSA is structured so both aerobic and linear speed development are emphasised in conjunction with the team's competition demands and the individual capabilities of the player.

\section{Acknowledgements}

We would like to thank the players and staff at the Football Association of Ireland for their hard work and cooperation throughout the study. 


\section{Disclosure Statement}

No potential conflict of interest was reported by the authors

\section{Funding}

Funding for this research was provided by the Institute of Technology Carlow and the Football Association of Ireland.

\section{REFERENCES}

[1] Gravina, L., Ruiz, F., Lekue, J.A., Irazusta, J. and Gil, S.M., 2011. Metabolic impact of a soccer match on female players. Journal of sports sciences, 29(12), pp.1345-1352.

[2] Silva, J.R., Rumpf, M.C., Hertzog, M., Castagna, C., Farooq, A., Girard, O. and Hader, K., 2018. Acute and residual soccer match-related fatigue: a systematic review and meta-analysis. Sports Medicine, 48(3), pp.539-583.

[3] Paul, D.J., Bradley, P.S. and Nassis, G.P., 2015. Factors affecting match running performance of elite soccer players: Shedding some light on the complexity. International journal of sports physiology and performance, 10(4), pp.516-519.

[4] Trewin, J., Meylan, C., Varley, M.C. and Cronin, J., 2018. The match-to-match variation of match-running in elite female soccer. Journal of science and medicine in sport, 21(2), pp.196-201.

[5] Hewitt, A., Norton, K. and Lyons, K., 2014. Movement profiles of elite women soccer players during international matches and the effect of opposition's team ranking. Journal of sports sciences, 32(20), pp.1874-1880.

[6] Datson, N., Drust, B., Weston, M. and Gregson, W., 2019. Repeated high-speed running in elite female soccer players during international competition. Science and Medicine in Football, 3(2), pp.150-156.

[7] Datson, N., Hulton, A., Andersson, H., Lewis, T., Weston, M., Drust, B. and Gregson, W., 2014. Applied physiology of female soccer: an update. Sports Medicine, 44(9), pp.1225-1240.

[8] Gabbett, T.J., Wiig, H. and Spencer, M., 2013. Repeated high-intensity running and sprinting in elite women's soccer competition. International Journal of Sports Physiology and Performance, 8(2), pp.130-138

[9] Datson, N., Drust, B., Weston, M., Jarman, I.H., Lisboa, P.J. and Gregson, W., 2017. Match physical performance of elite female soccer players during international competition. The Journal of Strength \& Conditioning Research, 31(9), pp.2379-2387.

[10] Ramos, G.P., Nakamura, F.Y., Penna, E.M., Wilke, C.F., Pereira, L.A., Loturco, I., Capelli, L., Mahseredjian, F., Silami-Garcia, E. and Coimbra, C.C., 2019. Activity Profiles in U17, U20, and Senior Women's Brazilian National Soccer Teams During International Competitions: Are There
Meaningful Differences? The Journal of Strength \& Conditioning Research, 33(12), pp.3414-3422.

[11] Nakamura, F.Y., Pereira, L.A., Loturco, I., Rosseti, M., Moura, F.A. and Bradley, P.S., 2017. Repeated-sprint sequences during female soccer matches using fixed and individual speed thresholds. The Journal of Strength \& Conditioning Research, 31(7), pp.1802-1810

[12] Andersson, H.Å., Randers, M.B., Heiner-Møller, A., Krustrup, P. and Mohr, M., 2010. Elite female soccer players perform more high-intensity running when playing in international games compared with domestic league games. The Journal of Strength \& Conditioning Research, 24(4), pp.912-919.

[13] Mohr, M., Krustrup, P., Andersson, H., Kirkendal, D. and Bangsbo, J., 2008. Match activities of elite women soccer players at different performance levels. The Journal of Strength \& Conditioning Research, 22(2), pp.341-349

[14] Girard, O., Mendez-Villanueva, A. and Bishop, D., 2011. Repeated-sprint ability-Part I. Sports medicine, 41(8), pp.673-694.

[15] Bishop, D., Girard, O. and Mendez-Villanueva, A., 2011. Repeated-sprint ability-Part II. Sports medicine, 41(9), pp.741-756.

[16] Turner, A.N. and Stewart, P.F., 2013. Repeat sprint ability. Strength \& Conditioning Journal, 35(1), pp.37-41.

[17] Spencer, M., Bishop, D., Dawson, B. and Goodman, C., 2005. Physiological and metabolic responses of repeated-sprint activities. Sports Medicine, 35(12), pp.1025-1044.

[18] Idrizović, K. and Raičković, N., 2013. The correlation between aerobic power, acceleration, repeat-sprint and speed endurance in elite female football. Research in Physical Education, Sport \& Health, 2(2).

[19] Shalfawi, S., Enoksen, E. and Tonnessen, E., 2014. The relationship between measures of sprinting, aerobic fitness, and lower body strength and power in well-trained female soccer players. International Journal of Applied Sports Sciences, 26(1), pp.18-25

[20] Sanders, G.J., Turner, Z., Boos, B., Peacock, C.A., Peveler, W. and Lipping, A., 2017. Aerobic capacity is related to repeated sprint ability with sprint distances less than 40 meters. International Journal of Exercise Science, 10(2), p.197.

[21] Gabbett, T.J., 2010. The development of a test of repeated-sprint ability for elite women's soccer players. The Journal of Strength \& Conditioning Research, 24(5), pp.1191-1194.

[22] Gabbett, T.J. and Mulvey, M.J., 2008. Time-motion analysis of small-sided training games and competition in elite women soccer players. The Journal of Strength \& Conditioning Research, 22(2), pp.543-552.

[23] Lockie, R.G., Liu, T.M., Stage, A.A., Lazar, A., Giuliano, D.V., Hurley, J.M., Torne, I.A., Beiley, M.D., Birmingham-Babauta, S.A., Stokes, J.J. and Risso, F.G., 2018. Assessing Repeated-Sprint Ability in Division I Collegiate Women Soccer Players. J Strength Cond Res, 26.

[24] Pardos-Mainer, E., Casajús, J.A. and Gonzalo-Skok, O., 
2019. Reliability and sensitivity of jumping, linear sprinting and change of direction ability tests in adolescent female football players. Science and Medicine in Football, 3(3), pp.183-190.

[25] Krustrup, P., Mohr, M., Amstrup, T., Rysgaard, T., Johansen, J., Steensberg, A., Pedersen, P.K. and Bangsbo, J., 2003. The yo-yo intermittent recovery test: physiological response, reliability, and validity. Medicine \& Science in Sports \& Exercise, 35(4), pp.697-705.

[26] Grgic, J., Oppici, L., Mikulic, P., Bangsbo, J., Krustrup, P. and Pedisic, Z., 2019. Test-Retest Reliability of the Yo-Yo Test: A Systematic Review. Sports Medicine, pp.1-11

[27] Hopkins, W.G. and Hopkins, W., 2009. A scale of magnitude for effect statistics. In: A NewView of Statistics, 2009, https://www.ugr.es/ fmocan/MATERIALES\%20DO
CTORADO/Progressive\%20Statistics\%20for\%20Studies\% 20in\%20Sports.pdf , Accessed 29/05/2020

[28] Cohen, J., 1988. Statistical Power Analysis for the Behavioural Sciences-Second Edition. 12 Lawrence Erlbaum Associates Inc. Hillsdale, New Jersey, 13.

[29] Glaister, M., Howatson, G., Pattison, J.R. and McInnes, G., 2008. The reliability and validity of fatigue measures during multiple-sprint work: an issue revisited. The Journal of Strength \& Conditioning Research, 22(5), pp.1597-1601.

[30] Passos Ramos, G., Datson, N., Mahseredjian, F., Lopes, T.R., Coimbra, C.C., Prado, L.S., Nakamura, F.Y. and Penna, E.M., 2019. Activity profile of training and matches in Brazilian Olympic female soccer team. Science and Medicine in Football, 3(3), pp.231-237. 